\title{
Orientational Selectivity of Hot Electrons Generated by a Dimer of Plasmonic
} Nanoparticles

\section{Supporting Information}

\author{
Maxime S. Maurice, ${ }^{*}$ Noémi Barros, ${ }^{*}$ and Hamid Kachkachi* \\ Université de Perpignan Via Domitia and Laboratoire PROMES-CNRS (UPR 8521), \\ Rambla de la Thermodynamique, Tecnosud, 66100 Perpignan, FRANCE. \\ E-mail: maxime.maurice@promes.cnrs.fr; noemi.barros@univ-perp.fr; \\ hamid.kachkachi@univ-perp.fr
}




\section{Permittivities for bulk silver and silver nanoparticles}

Fig. S1, S2 and S3 provide details about silver permittivities in bulk and NP, the frequency dependence of the parameters $\varepsilon_{\infty}^{\text {bulk }}$ and $\tau_{p}$, the cross-section of a NP in air, and the consequence of finite-size effects on these curves.
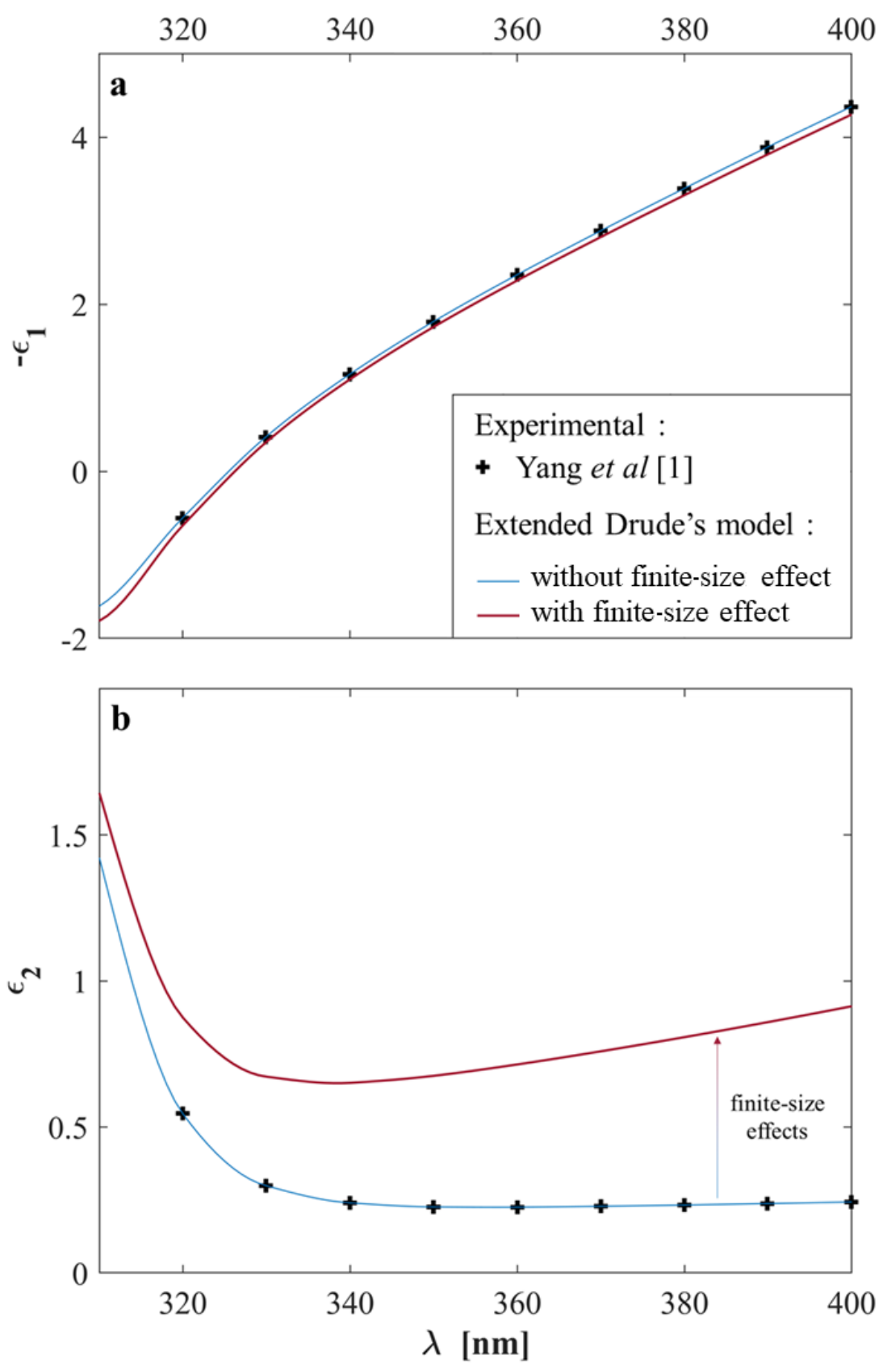

Figure S1: Dielectric permittivity of silver : bulk and finite-size effects. Real part (a) and imaginary part (b) of silver permittivity. Black crosses are experimental results for bulk silver from Ref. ${ }^{1}$. The blue curve is obtained using the extended Drude's model of Eq. 1 based on the experimental results : $\varepsilon^{\text {bulk }}$. The red curve is the permittivity including finite-size effects using Eqs. 2 and $3: \varepsilon^{\mathrm{NP}}$. We set $D=5 \mathrm{~nm}$ and $A=0.7$. 

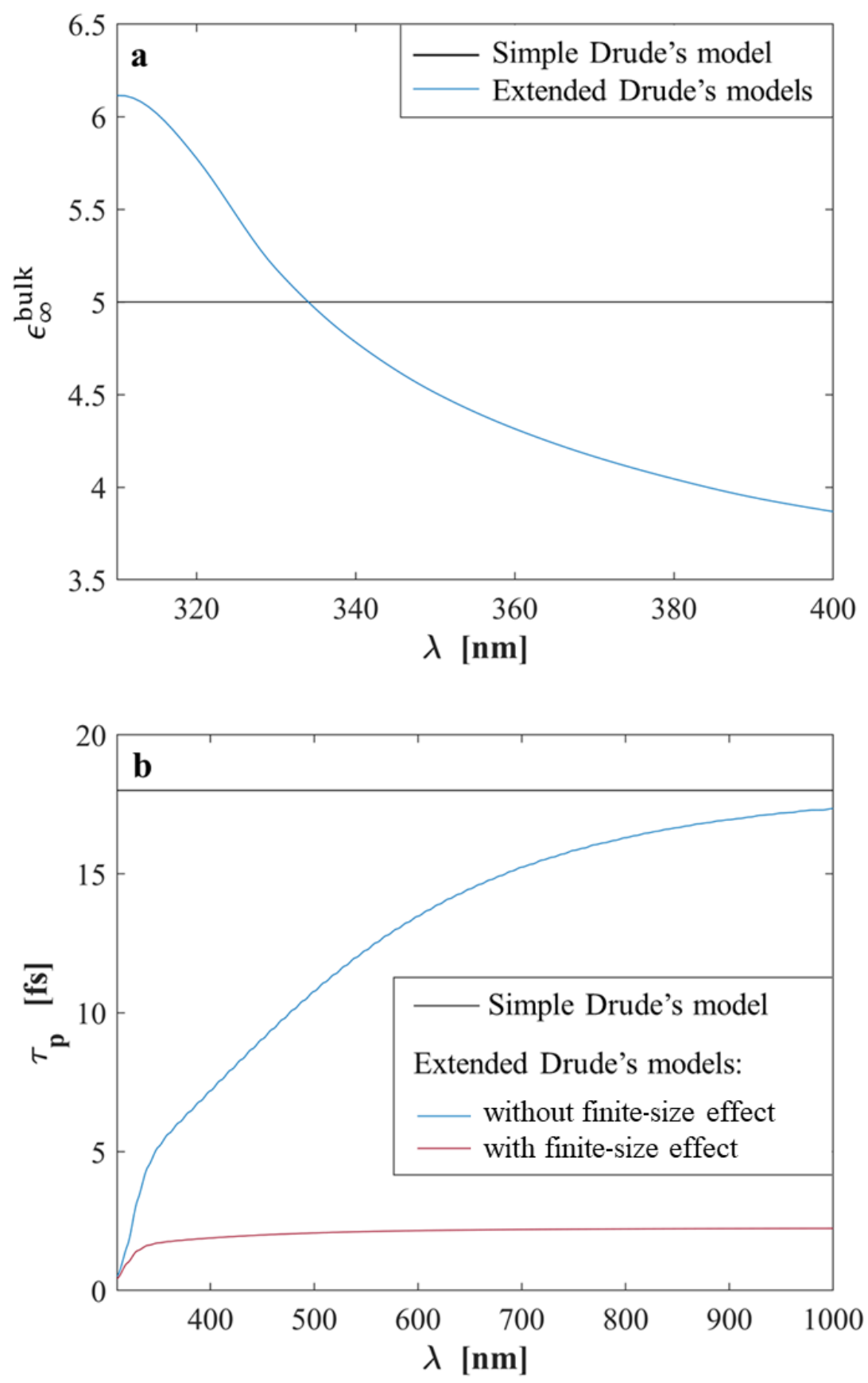

Figure S2: Background permittivity and plasmon lifetime spectral dependencies. (a) Background permittivity obtained with the simple Drude's model in ${ }^{1}$ (black curve) and the extended Drude's model of Eq. 1 (blue curve), fitted from the experimental data of Ref. ${ }^{1}$. (b) Plasmon lifetime obtained for the bulk with the simple Drude's model (black curve) and the extended Drude's model of Eq. 1 (blue curve), fitted from the experimental data of Ref. ${ }^{1}$. The red curve represents the plasmon lifetime for the NP including finite-size effects calculated using Eqs. 2 and 3 (red curve). The last values are used in our model. We set $D=5 \mathrm{~nm}$ and $A=0.7$. 


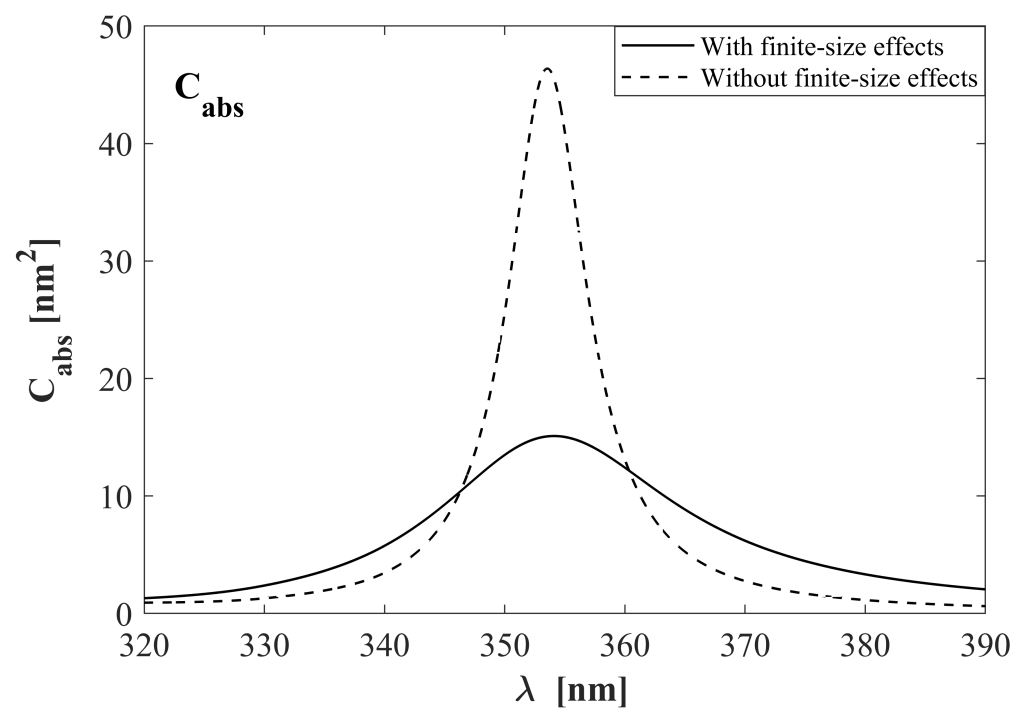

Figure S3: Calculated absorption cross-section for a spherical silver NP with $\mathbf{5} \mathbf{~ n m}$ diameter in air. The cross-section has been computed using the Clausius-Mossoti expression. The dotted curve is obtained using Eq. 1 for the permittivity ; the plain curve curve is obtained using Eq. 3 for the permittivity.

\section{Convergence criteria for the numerical integration}

In our calculations, the following criteria were used:

1) In the semi-analytical solution of Schrödinger equation, the energies were converged at $1 \mathrm{E}-5$ (in atomics units); the potential $V_{0}$ was adjusted in order to obtain a work function of $4.500 \pm 0.001 \mathrm{eV}$

2) For the dimer, the optical potential is numerically computed using the toolbox MNPBEM based on a boundary element method to solve Maxwell's equations. For this, the surface of each NP is modeled as a set of facets. We investigated the effect of the number of facets on the amplitude of the plasmon potential, and chose to work with 400 facets, as this ensures an error smaller than $1 \%$ at moderate computational cost.

3) For the computation of the coupling integrals appearing in Eq. (6), the effect of the cutoff radius was also investigated. We chose to use a cut-off of $\mathrm{R}+1 \mathrm{~nm}$, as this ensures an error 
smaller than $0.1 \%$ for each integral and on the total generation rates at moderate computational cost.

4) When using the adaptive quadrature scheme, the default parameters used by MATLAB for the threshold have been used.

5) Concerning the trapezoïdal integration, the effect of meshing has first been investigated for the isolated particle. If the optical potential is computed numerically using MNPBEM with the criteria defined in point 2 , and the integrals are calculated using a polar mesh of $110 \times 155$ points in the yz plane, then the error in each integral amplitude, and in the total generation rate, with respect with the reference calculation (analytical potential and wave functions + adaptive quadrature scheme), is found to be lower than $3 \%$. The energy distribution obtained using both numerical procedures is given in Fig. S4. 

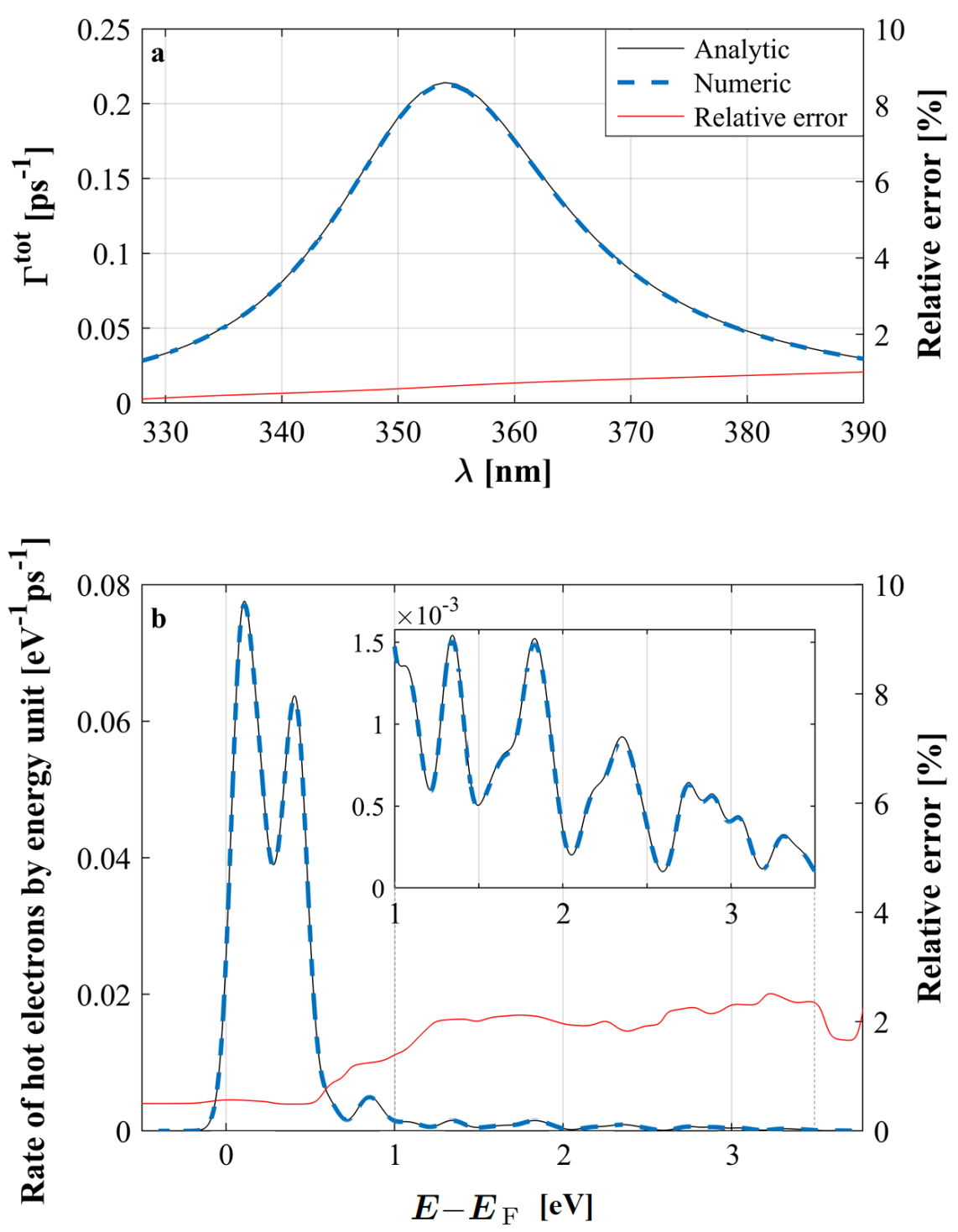

Figure S4: Comparison of our two integration approaches to compute the hot electrons generation rates in the case of an isolated NP. "Analytical": the optical potential is computed analytically and the $M_{i j}$ terms (Eq. 6) are computed using an adaptative quadrature scheme.

"Numerical": the optical potential is computed on a polar mesh of $110 \mathrm{x} 155$ points in the $y O z$ plane centered on one NP, and the $M_{i j}$ terms are computed using a trapezoidal integration. In each figure, the black solid lines, the blue dashed lines and the red solid line refer respectivelly to the analytic description, to the numeric description, and to the relative error in percent beetwen them. (a) Total hot electrons generation rate $\Gamma^{\text {tot }}(\omega)$. (b) Energy distribution at the resonance wavelength $(354 \mathrm{~nm})$. Inset: zoom in the region 1-3.6 eV above the Fermi energy $E_{\mathrm{F}}$.

6) For the dimer, the accuracy of the grid has then been re-evaluated by studying the convergence of the integrals with the number of points, see for instance Fig. S5. The results confirm that the mesh is fine enough to ensure a convergence to within $0.5 \%$ in the total generation rates, 
the average energy of the hot electrons and the spill-out parameter.
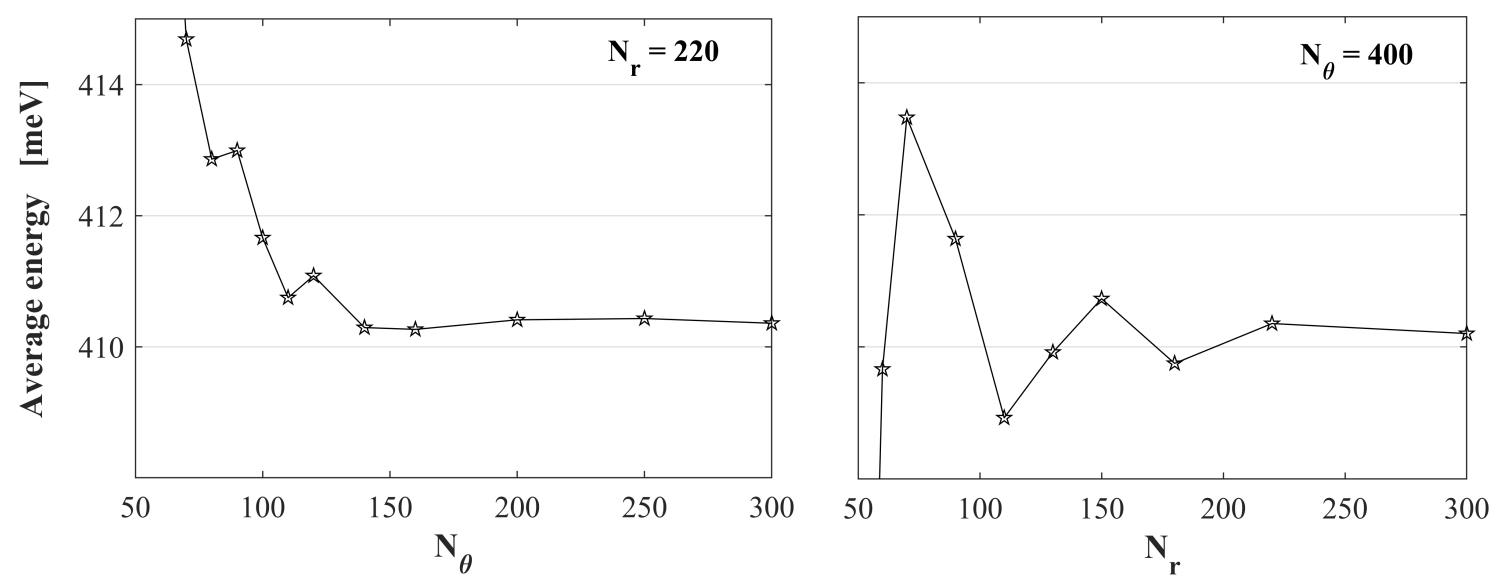

Figure S5: Example of convergence studies for the trapezoïdal integration scheme : effect of the number of points in the integration mesh on the average energy of the generated hot electrons. Using the chosen grid with $110 \times 155$ points, the relative error on this energy compared to the finest possible grid is lower than $0.5 \%$. The system investigated here is the dimer of NPs with a gap distance of $1 \mathrm{~nm}$, at the resonance wavelength.

\section{References}

(1) Yang, H. U.; D’Archangel, J.; Sundheimer, M. L.; Tucker, E.; Boreman, G. D.; Raschke, M. B. Optical dielectric function of silver. Phys. Rev. B 2015, 91, 1-11. 